\title{
Cultura y marketing en las bibliotecas públicas españolas: actividades, iniciativas y recursos
}

\author{
Por José Luis Herrera Morillas y Margarita Pérez Pulido
}

\begin{abstract}
Resumen: Hoy es imprescindible que las bibliotecas promuevan el uso de sus servicios, se den a conocer y atraigan a los lectores. Este artículo analiza la difusión cultural y el marketing en las bibliotecas públicas españolas. Se consultan 183 sitios web. Se recopilan las actividades, iniciativas y recursos. Se sistematizan, cuantifican y describen.

Palabras clave: Cultura, Marketing, Bibliotecas públicas, Servicios bibliotecarios, Promoción.

Title: Culture and marketing in Spanish public libraries: activities, initiatives and resources.

Abstract: Today it is essential for libraries to promote the use of their services, make themselves be known and attract readers. This article discusses the cultural dissemination and marketing in Spanish public libraries, based on an analysis of 183 web sites. Information was gathered on activities, initiatives and resources that were then systematized, quantified and described.
\end{abstract}

Keywords: Culture, Marketing, Public libraries, Library services, Promotion.

Herrera Morillas, José Luis; Pérez Pulido, Margarita. "Cultura y marketing en las bibliotecas públicas españolas: actividades, iniciativas y recursos". En: El profesional de la información, 2007, enero-febrero, v. 16, n. 1, pp. 62-76.

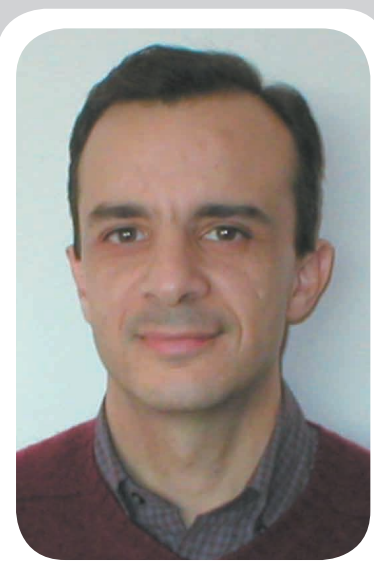

José Luis Herrera Morillas, doctor en documentación por la Universidad de Granada profesor de la Facultad de Biblioteconomía y Documentación de la Universidad de Extremadura, entre sus últimos trabajos publicados "Los elementos de identidad visual corporativa de las bibliotecas universitarias españolas proyectados a través de internet".

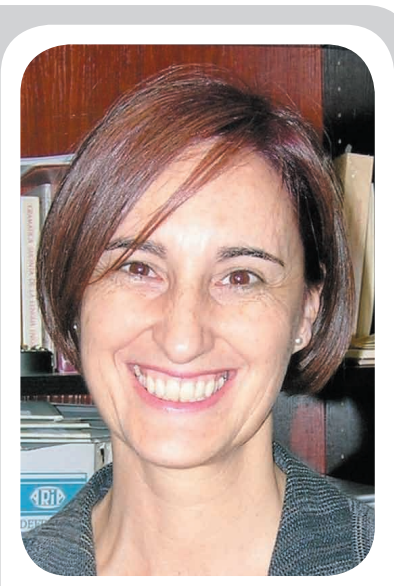

Margarita Pérez Pulido, doctora en Documentación por la Universidad de Salamanca, es profesora titular de escuela en la Facultad de Biblioteconomía y Documentación de la Universidad de Extremadura, imparte las asignaturas Organización y administración de bibliotecas y Planificación y evaluación de unidades de información. Entre sus últimas publicaciones citamos el libro "Teoría y nuevos escenarios de la biblioteconomía". http://dx.doi.org/10.3145/epi.2007.ene.08

\section{Introducción}

ESTE TRABAJO HA CONSISTIDO en comprobar qué actividades, iniciativas $y$ recursos relacionados con la difusión cultural y el marketing ofrecen las bibliotecas públicas españolas, que son aspectos que consideramos esenciales para establecer vínculos entre la biblioteca y la comunidad, despertar interés por ella y promocionarla.

La realización de actividades culturales en las bibliotecas cuenta con gran tradición, formando parte de la denominada "extensión cultural", un medio de introducir el libro en el ámbito de la cultura (Caballero Garrido, 2002). La aplicación del marketing es más reciente, se extiende a partir del último tercio del siglo XX. Hasta ese momento las actividades culturales servían de promoción y difusión bibliotecaria. En las bibliotecas españolas (como hemos comprobado con este trabajo) las actividades culturales están más extendidas que las de marketing, aunque éstas también se desarrollan continuamente.

\section{"En las bibliotecas espa- ñolas las actividades cul- turales están más extendi- das que las de marketing"}

Las fuentes de estudio utilizadas han sido los sitios web de las bibliotecas, medios eficaces para obtener información de manera rápida y actualizada, ya que permiten conseguir una visión de conjunto de un buen número de bibliotecas en un período de tiempo reducido. Sin embargo somos conscientes de que existen centros que no informan de sus actividades y servicios a través de este canal y sí que realizan actividades de este tipo. De todos modos, hemos adoptado este criterio por considerar que las bibliotecas con web constituyen una muestra representativa para el estudio, y además supone una ventaja añadida al reducir el tiempo dedicado a la localización y recogida de los datos (asunto importante en un estudio de estas características que quiere mostrar un estado de la cuestión reciente).

El empleo del marketing al servicio de las bibliotecas se remonta a 1970 (Norman, 1992) y se fundamenta en la concepción de la 


\section{Sitios web de las bibliotecas citadas}

- Biblioteca d'Alcúdia Can Torró (Mallorca). http://www.cantorro.es/principal/index. php? $i=e s p$

- Biblioteca Central de Igualada (Barcelona). http://www.aj-igualada.net/editables/ajuntament/cultura/biblioteca/biblioteca.htm

- Biblioteca Infantil de Can Butjosa (Barcelona).

http://bibut.parets.org/

- Biblioteca Joan Oliva i Milà de Villanova i la Geltrú (Barcelona).

http://www.vilanova.cat/biblioteca/Caste Ilano/Presentacion. htm

- Biblioteca Joan Triadú (Vic).

http://www.bibliotecavic.com/html/homebanners.htm

- Biblioteca del Mil.lenari, SantCugat del Vallés.

http://www.biblioteca.santcugat.org/

- Biblioteca de Moià (Barcelona).

http://moia.nautes.org/informativ/biblio/index.htm

- Biblioteca Municipal de Albaida (Valencia)

http://www.albaida.org/val/biblioteca.asp

- Biblioteca Municipal de Cassà de la Selva (Gerona).

http://www.salagala.com/

- Biblioteca Municipal de La Palma de Cervelló (Barcelona).

http://www.bibliolapalma.cat/

- Biblioteca Municipal de La Rambla (Córdoba).

http://www.biblioteca.aytolarambla.org/

- Biblioteca Municipal de Tossa "Manuel Vilà i Dalmau" (Gerona).

http://www.ddgi.es/bibtossa/

- Biblioteca Pública Barañain (Navarra).

http://www. bibliotecaspublicas.es/baranain/index.jsp

- Biblioteca Pública Central en Vigo.

hitp://www.rbgalicia.org/bpvi/

- BPE de Almería "Francisco Villaespesa".

http://www.juntadeandalucia.es/cultura/

opencms/export/bibliotecas/bibalmeria/index.html

- BPE de Badajoz "Bartolomé J. Gallardo". http://www.bibliotecaspublicas.es/badajoz/index.jsp

BPE de Burgos.

http://www.bibliotecaspublicas.es/burgos/ index.jsp

- BPE de Ciudad Real.

http://www. bibliotecaspublicas.es/ciudadreal/index.jsp

- BPE de Cuenca "Fermín Caballero".

http://www.bibliotecaspublicas.es/cuenca/ index.jsp

- BPE de Granada.

http://www.juntadeandalucia.es/cultura/ opencms/export/bibliotecas/bibgranada/index.html

- BPE de Guadalajara.

http://www.bibliotecaspublicas.es/guadalajara/index.jsp

- BPE de Lérida.

hHtp://cultura.gencat.net/bpl/

- BPE de Lugo.

hHtp://www.rbgalicia.org/bplu/

- BPE de Málaga.

http://www.juntadeandalucia.es/cultura/ opencms/export/bibliotecas/bibmalaga/ - BPE de Mérida "Jesús Delgado Valhondo". http://www.bibliotecaspublicas.es/merida/ index.jsp

- BPE de Oviedo.

http://www.bibliotecaspublicas.es/oviedo/ index.jsp

- BPE de Salamanca.

http://www.bibliotecaspublicas.es/salaman$\mathrm{ca} /$ index.jsp

- BPE de Sevilla.

http://www.juntadeandalucia.es/cultura/ opencms/export/bibliotecas/bibsevilla/index.html

BPE de Tarragona.

http://194.179.95.78/bpt/cs/index.htm

- BPE de Valencia.

http://www.gva.es/bpv/indice.htm

BPE de Valladolid.

http://bpval.bcl.jcyl.es/

- BPE de Zamora.

http://www.bibliotecaspublicas.es/zamora/ index.jsp

BPE de Zaragoza.

http://portal.aragob.es/servlet/page? pageid $=4518 \&$ dad $=$ portal $30 \&$ schema $=P O R T A L 30 \&$ _type $=$ site\&_fsite $i d=867 \&$ fid $=1303375 \&$ fnavba rid $=1326823 \&$ fnavbarsiteid $=867 \&$ \&edit $=0 \& \_$mode $=2 \&$ f fdisplaymode $=1$ \&_fcalledfrom $=1$ \&_fdisplayurl $=$

- Biblioteca Pública de Moaña (Pontevedra).

http://moana.servidores.net/

- Biblioteca Pública Municipal de Armilla (Granada).

http://bmparmilla.iespana.es/

- Biblioteca Pública Municipal de Avilés (Asturias).

http://www.bibliotecaspublicas.es/aviles/index.jsp

- Biblioteca Pública Municipal de Bembibre (León).

http://www. bibliotecaspublicas.es/bembibre/index.jsp

- Biblioteca Pública Municipal de Burriana (Castellón).

http://www. bibliotecaspublicas.es/burriana/index.jsp

- Biblioteca Pública Municipal de Candás "Carlos González Posada" (Asturias).

hitp://www.bibliotecaspublicas.es/candas/ index.jsp

- Biblioteca Pública Municipal de Castuera "Gonzalo Soubrier" (Badajoz).

http://www.biblio-castuera.com/

- Biblioteca Pública Municipal de Coaña "Gonzalo Anes" (Asturias).

http://www.bibliotecaspublicas.es/coana/ index.jsp

Biblioteca Pública Municipal de Dúrcal (Granada).

http://www.adurcal.com/servicios/bibliote$\mathrm{ca} /$ index.htm

Biblioteca Pública Municipal de Grado "Valentín Andrés Álvarez" (Asturias).

http://www.bibliotecaspublicas.es/grado/ index.jsp

- Biblioteca Pública Municipal de La Pola Lena "Menéndez Pidal" (Asturias).

http://www.bibliotecaspublicas.es/poladelenaa/index.jsp

- Biblioteca Pública Municipal de Peñaranda de Bracamonte (Salamanca).

http://www.fundaciongsr.es/penaranda/ biblioteca/dosframes.htm

- Biblioteca Pública Municipal de Pravia "Antón de la Braña" (Asturias).

http://www.bibliotecaspublicas.es/pravia/ index.jsp

- Biblioteca Pública Municipal de San Martín del Rey Aurelio (Asturias).

http://www.bibliotecaspublicas.es/sanmartin/index.jsp

- Biblioteca Pública Municipal de Villajoyosa "Cristóbal Zaragoza" (Alicante).

http://biblioteca.villajoyosa.com/

- Biblioteca Pública Salvador Raurich de Begur (Gerona).

http://www.begur.org/Biblioteca.htm

- Biblioteca Pública de Pamplona Yamaguchi. http://www.bibliotecaspublicas.es/pamplona/index.jsp

- Biblioteca Pública de Roses "Jaume Vicens Vives".

http://www.bibgirona.net/roses/

- Bibliotecas de Barcelona.

http://www.bcn.es/icub/biblioteques/

- Bibliotecas Municipales de Lorca (Murcia).

http://bibliotecasmunicipales. lorca.es/presentacion.asp

- Bibliotecas Públicas Municipales de Madrid. http://gestiona.madrid.org/bpcm/lared.html - Red de Bibliotecas de Alcoy (Valencia).

http://www.ajualcoi.org/wbiblioteca/val/ index.htm

- Red de Bibliotecas de la Diputación de Barcelona.

http://www.diba.es/biblioteques/

Red de Bibliotecas Municipales de Bilbao. http://www.bilbao.net/Bibliotecas/isp/pwebioOO.jsp?idioma $=c$

- Red de Bibliotecas Municipales de Cartagena (Murcia).

http://www.cartagena.es/bibcartagena/

- Red de Bibliotecas Públicas de Ávila.

http: / / www. avila.es/Principal/Servicios\% 20 Municipales/ Cultura\%20y\%20Deportes/Bibliotecas/

- Red de Bibliotecas Públicas del Ayuntamiento de Oviedo.

http://www4.ayto-oviedo.es/es/areasMunicipales/educacion/bibliotecas/bibliotecas. cipal

- Red de Bibliotecas Públicas de Mieres (Asturias).

http://www.bibliotecaspublicas.es/mieres/ index.jsp

- Red de Bibliotecas Públicas Municipales de Salamanca.

http://bibliotecas.ayłosalamanca.es/

- Red de Bibliotecas Públicas Municipales de Siero (Asturias).

http://www. bibliotecaspublicas.es/siero/index.jsp

- Red Municipal de Bibliotecas de Murcia.

http://rmbm.org/

- Red Municipal de Bibliotecas de Sevilla

http://www.ingesnet.com/rbmsevilla/mapa. asp

- Servicio Municipal de Bibliotecas de Córdoba.

http://biblioteca.ayuncordoba.es/

- Servicio Municipal de Bibliotecas Públicas de San Javier (Murcia).

http://biblioteca.sanjavier.es/ biblioteca como organización de servicios que implica ponerla en relación con el mercado, necesidad derivada por el hecho de que se sitúa en un entorno competitivo con otros servicios culturales e infor- mativos y debe afirmarse frente a ellos, lograr su cuota de mercado, de uso, lo que implica introducir en el proceso de gestión bibliotecaria la filosofía de que el usuario es lo primero, un cliente al que hay que captar, satisfacer y mantener (Gómez Hernández, 1997).

De este modo hoy es imprescindible que se promueva el uso de sus servicios, se den a conocer 
y atraigan a los lectores para que efectivamente lo sean utilizando las técnicas del marketing. Pero, además, por ser piezas clave de la infraestructura cultural de la comunidad (todas en general, pero especialmente las públicas por el amplio espectro de usuarios al que van dirigidas), deben seguir dando prioridad a la organización de actividades culturales, lo que se denomina en biblioteconomía la "extensión cultural", consistente en colaborar y promover la vida cultural en general de su ámbito, excediendo lo que es la mera promoción de sus colecciones (Gómez Hernández, 2002). De este modo, toda biblioteca y especialmente la pública debe diversificar y ampliar sus funciones tradicionales de consulta y préstamo abriéndose a la sociedad mediante el fomento de actividades culturales, "sirviendo como lugar de encuentro de la comunidad" (García Rodríguez, 1996) y colaborando de este modo en la promoción y atracción ejercida a través de las actividades de marketing.

\section{Metodología}

El trabajo se centra en el análisis de las 183 webs de bibliotecas públicas españolas reunidas en el sitio "Red de sedes web de las bibliotecas públicas"1 del Ministerio de Cultura. Como trabajo previo revisamos varias tipologías recientes sobre actividades y actuaciones culturales y de marketing en el entorno de las bibliotecas, debido a que la delimitación no es nítida y es evidente que detrás de muchas actividades culturales hay un plan de marketing; al igual que muchas acciones culturales lo son de marketing. Quílez (2002) afirma al respecto: "A pesar de la notoriedad despertada por la realización de las actividades culturales en las bibliotecas públicas, queda mucho por hacer con respecto a la normalización de su gestión que se ve dificultada por la diversidad de formas y contenidos, por el escaso número de estudios sobre el particular y por una concepción tendente todavía a considerarlas ornamento de la oferta de los centros".

Las tipologías consultadas son las siguientes:

Directrices IFLA/Unesco para el desarrollo del servicio de bibliotecas públicas (2002) en el apartado dedicado al "Plan de promoción y comercialización" incluye actividades como:

—Exposiciones y presentaciones.

-Publicaciones periódicas y preparación de listas de recursos y folletos.

- Campañas de lectura y alfabetización.

- Concepción de campañas para responder a las necesidades de personas que tienen alguna discapacidad física o sensorial.

—Ferias de libros.

— Sitios de la biblioteca en la Red.

-Grupos de amigos de la biblioteca.

- Celebraciones anuales de la semana de la biblioteca y otras actividades colectivas de promoción.

—Celebración de años especiales y aniversarios.

—Listín de la biblioteca en la guía telefónica y otros repertorios comunitarios.

-Actividades y campañas de recaudación de fondos.

—Publicaciones especiales sobre la biblioteca, por ejemplo, historia de la biblioteca o de la comunidad.

Las Pautas Calimera de buena práctica (2005) en su epígrafe "Publicidad, marketing y extensión", enumera estas actuaciones:

-Boletín de noticias.

- Enviar publicidad por correo a los posibles usuarios del servicio.
—Confección y distribución de folletos.

- Visitas a las empresas, centros educativos, asociaciones, etc., de la localidad.

-Participación activa del personal y directores de los archivos, bibliotecas y museos en foros y debates públicos, así como en periódicos y programas de TV y radio.

Las Pautas Pulman (2002) consideran como actividades culturales: charlas, conciertos y lecturas poéticas. También recogen un apartado denominado "Publicidad, marketing y extensión bibliotecaria" en el que recomienda a la biblioteca que dé publicidad de forma continuada a lo largo del tiempo, a los servicios y recursos que allí se ofrecen. Y para ello debe:

- Enviar publicidad por correo a los posibles usuarios del servicio.

-Preparar folletos.

—Exponer información detallada en el servidor web de la biblioteca.

- Publicar listados de fondos bibliográficos.

- Realizar visitas a las empresas, centros educativos, asociaciones, etc., de la localidad.

-Elaborar un boletín de noticias.

La Pautas sobre los servicios de las bibliotecas públicas (2002) establecen que se deben organizar actividades culturales que fomenten y refuercen el uso y conocimiento de la entidad como centro cultural, informativo y lúdico, así como su vocación de espacio ciudadano colectivo, para lo cual organizarán, directamente o contando con la colaboración y apoyo de otras entidades locales:

- Hábito de la lectura entre el público adulto.

-Idem entre niños y jóvenes: la hora del cuento, maratones de cuenta 
cuentos, actividades prácticas de escritura ilustración y edición de los propios libros o publicaciones periódicas, concursos, talleres de lectura, encuentros con autores e ilustradores.

-Exposiciones bibliográficas, artísticas, científicas, divulgativas, etc., en las que se prestará una especial atención a los temas de interés local.

-Conferencias, mesas redondas, recitales y veladas literarias, encuentros con autores, ilustradores, editores y libreros, presentaciones de libros y publicaciones en cualquier soporte.

- Narración oral, a fin de preservar y difundir las culturas, la historia y tradiciones de la comunidad local.

-Otras iniciativas que impulsen las manifestaciones culturales locales.

Quílez (2002) establece que en las bibliotecas públicas se ha pasado de concebir esas tareas como mero adorno ocasional del centro, o como algo disociado de la biblioteca, a valorarlas en diversos aspectos: como complemento a la labor informativa y difusora de la cultura de las bibliotecas (extensión cultural); como instrumento de la difusión de fondos y promoción de servicios o como medio para la inclusión en la vida social y cultural de la ciudad (presencia en los medios de comunicación, reclamo para no usuarios). A partir de este planteamiento define un modelo de clasificación de las actividades en dos niveles de detalle: categorías, según la finalidad perseguida con la actividad, y tipos, dependiendo del formato en que se presentan. Las cuatro categorías propuestas son:

—Extensión cultural.

-Fomento de la lectura.

—Formación de usuarios.

-Promoción de fondos y servicios.
Nos interesan para nuestro trabajo la primera y la cuarta. En la "Extensión cultural" integra las actividades realizadas para difundir aspectos de la cultura que no tengan una relación directa con los servicios de la biblioteca o con los fondos de que dispone (exposiciones, cursos, artes audiovisuales, conferencias, celebraciones especiales). Las actividades de "Promoción de fondos y servicios" persiguen que el usuario tenga presente la existencia de la variedad de fondos y servicios que le ofrece el centro (exposición de fondos, publicidad genérica, boletín informativo de novedades, panel/tablón de anuncios, web).

García Rodríguez (1996) mantiene que las actividades culturales realizadas por las bibliotecas pueden ser puntuales o permanentes, promovidas o asumidas por ella, y tienen como marco las propias instalaciones bibliotecarias u otras instalaciones con el objetivo de satisfacer las necesidades y deseos de información de sus clientes mediante servicios diferentes a los tradicionales servicios mínimos bibliotecarios. Para la citada autora las actividades más habituales son:

-Exposiciones.

-Mesas redondas y debates.

-Presentaciones de obras o colecciones.

-Representaciones teatrales.

-Audiciones musicales.

-Proyecciones cinematográficas.

-Conferencias.

Massísimo (2005) ha realizado un estudio en el que establece las cualidades que caracterizan al marketing bibliotecario:

—Diseño de productos a medida para segmentos específicos de usuarios (bibliomercados para las amas de casa; bibliopiscinas, biblioplayas y todas sus variantes para las personas de vacaciones; bibliometro para los que se desplazan en transporte público a sus lugares de trabajo; cursos para manejar internet; rincón de padres en la bebeteca (para los padres y abuelos encargados del cuidado de los niños).

-Inclusión de la promoción como parte del marketing-mix: uso de publicidad para dar a conocer estos servicios, desde los clásicos soportes impresos en diferentes variantes (folletos de mano, carteles, etc.) hasta otros objetos como marcapáginas, camisetas, etc.

-El logotipo como elemento identificativo de la imagen de la biblioteca y como mecanismo de promoción, así como para la búsqueda de nuevos públicos.

—Relaciones con el público y búsqueda de nuevos lectores: en el pasado la relación de la biblioteca con el usuario era sobre todo unidireccional, por ejemplo con folletos sobre ella misma o sus servicios, las guías de lectura, etc. Posteriormente (a mediados de los noventa) se comienzan a editar boletines informativos periódicos, que se pueden concebir como una forma más de prensa local. También se han marcado como objetivo buscar y fidelizar a nuevos públicos y para ello han puesto en marcha acciones que se celebran anualmente, como por ejemplo los "Itinerarios literarios" del Consorcio de Bibliotecas de Barcelona.

Siguiendo con los aspectos metodológicos, en una siguiente fase realizamos una exploración inicial de sus sitios web y comprobamos que la realidad que nos muestran las bibliotecas españolas está más cercana a los planteamientos de Quílez (2002) y a los de Massísimo (2005). De esta forma decidimos reunir los tipos de actividades que con más frecuencia se organizan en dos grupos, por un lado las de carácter cultural (coincide con lo que Quílez denomina "Extensión cultural") y por otro las más relaciona- 
das con la promoción y el marketing (siguiendo el esquema trazado por Massísimo). De todos modos (y como ya hemos mencionado) algunas de estas acciones pueden considerase a la vez dentro de cualquiera de esas dos categorías. Nosotros para agruparlas en una u otra hemos partido de la explicación de los autores citados y también en los casos más ambiguos hemos intentado dilucidar qué aspecto tiene más peso.

a. Actividades culturales:

\section{—Exposiciones.}

-Charlas y conferencias.

-Actividades varias con motivo de acontecimientos, conmemoraciones, celebraciones, etc.

-Certámenes, concursos y premios.

- Audiciones y actividades en torno a la música.

-Cine: cine forum, proyecciones y otras actividades alrededor del séptimo arte.

-Presentaciones de libros.

-Encuentros con autores.

- Tertulias, coloquios y debates.

-Poesía.

- Teatro.

—Feria del libro.

-Monográficos.

b. Actividades de promoción y marketing:

—Visitas guiadas o en grupo.

—Talleres.

-Bibliopiscinas.

—Concursos de marcapáginas y pegatinas.

-Bebetecas.

- Programas y actividades en coordinación con otras instituciones.

-Bibliotecarios por un día.

-Magia.

—Programas en la radio.
-Actos multiculturales o para inmigrantes.

—Bibliojardines.

- "Para conocer la ciudad".

- Tertulias o conversaciones en inglés.

- Tertulias literarias virtuales de escritores.

— "Ven y conoce tu biblioteca".

- Concursos para elegir la mascota de la biblioteca.

—“Jóvenes contra la violencia”.

— "Noches en la biblioteca".

— "Biblioviaje".

- Juegos de mesa en la biblioteca.

— "Biblioteca de la memoria".

- Semanas literarias.

—El rincón del juego.

—Club de los deberes.

-Encuentros de lectura (para los maestros).

—“Apadrinamiento".

— "Aventura de llegir".

— "Portes enllà" (Más allá de las puertas).

—Karaoke.

-Bibliocampamentos de verano.

— "Conócelos y aprende".

—_Biblioteca solidaria".

Durante la consulta de las webs hemos comprobado que algunas bibliotecas tienen disponibles recursos virtuales relacionados con el marketing y la difusión cultural. En este caso la oferta y variedad es menor si las comparamos con las actividades, por ello no hemos considerado necesario distinguir entre recursos de carácter cultural y de marketing. Son los siguientes:

- Novedades.

-Logotipos.

- Guías de lectura a texto completo.
—Rincón infantil o bebeteca, web infantil y juvenil, o web juvenil.

-Trípticos sobre servicios, actividades, etc., a texto completo.

-Formularios para solicitar visitas y otras actividades.

- Revistas o boletines de la biblioteca a texto completo.

—Reproducción de marcapáginas.

- Sección profesional (documentos para bibliotecarios).

-Planos de distribución de fondos y espacios.

—Guías del inmigrante.

—Catálogos de exposiciones.

- Textos informativos sobre autores locales.

—Recurso "El granadino del mes".

—Recurso "Sección de inéditos".

—Recurso "Sevilla literaria".

—Recurso "Así era".

—Recurso "Cartel de silencio".

-Buzón para devoluciones.

—Álbum de firmas.

-Formularios para seleccionar el tipo de actividad.

—Recurso "El rincón del lector".

-Recurso "Gente mayor en Tarragona".

\section{Resultados}

Los presentamos en tres grupos: actividades culturales, de marketing y recursos en línea relacionados con ambos aspectos. Se presentan en orden de frecuencia, indicando entre paréntesis el número de bibliotecas diferentes en los que los hemos localizado y se comentan las principales características utilizando las descripciones que aparecen en las webs, mencionando ejemplos concretos.

De las 183 web analizadas, en $113(61,7 \%)$ se informa sobre actividades culturales y/o de marke- 
ting; y en $104(56,8 \%)$ localizamos recursos relacionados.

\section{"En el $62 \%$ de las webs se informa sobre iniciativas culturales y/o de marke- ting; y el $57 \%$ dispone de recursos relacionados"}

\section{Actividades culturales}

-Exposiciones (54): se organizan tanto de fondos bibliográficos como de otros objetos. Así la Biblioteca Pública Municipal de Peñaranda de Bracamonte en el apartado "Exposiciones desde la biblioteca" explica que en ocasiones colabora con instituciones educativas o culturales en la organización de eventos relacionados con el libro o de marcado carácter informativo. Destaca la exposición itinerante "Libros troquelados" que recorrió trece pueblos de la provincia salmantina o el ciclo "Que veinte años no es nada...", organizado junto a los institutos de enseñanza secundaria de Peñaranda, cuyos primeros títulos fueron: La muerte de Franco (1995), Referéndum para la reforma política (1996), Primeras elecciones democráticas (1997)...

-Charlas y conferencias (30): la Red de Bibliotecas de la Diputación de Barcelona promueve conferencias literarias de autores destacados y de temas de actualidad.

-Actividades con motivo de acontecimientos, conmemoraciones, celebraciones, etc. (24): la $B P E$ de Valencia con motivo del Día internacional del libro infantil, y del bicentenario del nacimiento de Hans Christian Andersen, realizó actividades extraordinarias en horario de mañana y tarde. Los alumnos de literatura infantil de la Escuela Universitaria de magisterio Edetania realizan una sesión de animación a la lectura en la biblioteca, concertando cada año con distintos cursos de las escuelas de Valencia. Otro ejemplo lo tenemos en la Red de Bibliotecas Municipales de Murcia que coincidiendo con esa misma fecha realiza obsequios de libros a los usuarios.

-Certámenes, concursos y premios (23): recogemos una breve muestra:

a. Certamen de libros gigantes (BPE de Guadalajara): se convoca desde hace años entre los centros escolares de la ciudad para el Día del libro. Se les pide que participen todas las aulas del centro escolar y que el formato de presentación sea aproximadamente de un pliego de cartulina, que facilita el trabajo en equipo, pues cada clase puede encargarse de una de las páginas del libro y hay espacio suficiente para texto, ilustraciones, fotografías, etc. Es una actividad que familiariza al niño con el proceso de creación de un libro: texto, ilustración, encuadernación, etc. y desarrolla la creatividad y la expresión artística. Para el Día del libro se convoca un acto de presentación de todos los materiales recibidos y se entrega un lote de libros a cada centro para la biblioteca escolar. Más tarde, se hace una exposición de todos los trabajos presentados durante el Maratón de cuentos.

b. Certamen infantil de cuentos fantásticos (BPE de Ciudad Real).

c. Concurso "Locos por los libros" (Biblioteca Pública Municipal de Bembibre), consiste en averiguar, a través de un párrafo de un libro, el autor de éste y su título; puede participar todo aquel que quiera, sin límite de edad.

d. Concurso de "Microrrelatos" (BPE de Badajoz).

e. Concurso infantil de cuentos navideños (Red Municipal de Bibliotecas de Murcia). f. Concurso de postales de Navidad (Biblioteca Pública Municipal de Dúrcal).

g. Concursos de cómics, dibujo y creación literaria para niños y jóvenes (Red de Bibliotecas Públicas Municipales de Siero).

h. Premios a los superlectores del año (Red de Bibliotecas Publicas de Mieres).

- Audiciones y actividades en torno a la música (20): algunas de ellas son "Música en la biblioteca" (en colaboración con la Escuela Municipal de Música, Biblioteca Central de Igualada); "Ciclos de música" (Red de Bibliotecas de la Diputación de Barcelona); "El descubrimiento de la música" (Biblioteca Municipal de Tossa "Manuel Vilà i Dalmau"); o "Música entre libros" (BPE de Zaragoza).

- Cine, cine forum, proyecciones (20): las proyecciones se presentan como actividad destacada en la BPE de Granada. La Biblioteca Pública Municipal de Dúrcal (como otras muchas) organiza un cine club. La Red de Bibliotecas Públicas de Mieres es ejemplo de los centros que han optado por el cine forum, en este caso proyectando películas de los libros leídos. La BPE de Oviedo anuncia ciclos de cine. Finalmente citamos "Educando la mirada: introducción a los géneros cinematográficos" que se realiza en la Red Municipal de Bibliotecas de Murcia.

-Presentaciones de libros (20): la Red de Bibliotecas Públicas Municipales de Siero anuncia este tipo de evento dentro de las actividades de ocio; la BPE de Salamanca dentro de las actividades culturales; otro centro que las incluye es la Biblioteca Joan Oliva i Milà (Vilanova i la Geltrú).

-Encuentros con autores (13): la Red de Bibliotecas Municipales de Bilbao los ha diseñado de este 
modo: con el fin de contribuir al fomento del hábito lector entre los alumnos y de promocionar las bibliotecas municipales, se ofrece la posibilidad de participar en encuentros con escritores locales. Están dirigidos a alumnos de $5^{\circ}$ y $6^{\circ}$ de Primaria y todos los cursos de la ESO. En estos encuentros los estudiantes tratan en directo con los escritores más representativos de la literatura infantil y juvenil del entorno. Las sesiones, con 50 alumnos, se realizan en horario de mañana. Otras bibliotecas que han adoptado esta actividad son: la Red de Bibliotecas Públicas de Mieres, la BPE de Cuenca "Fermín Caballero", la $B P E$ de Guadalajara o la BPE de Lugo.

- Tertulias, coloquios y debates (13): dentro de un amplio abanico, gracias a esta oferta nos podemos encontrar desde tertulias con poetas (Biblioteca "Joan Triadú" de Vic); tertulias literarias (Biblioteca Pública de Pamplona Yamaguchi) hasta un café-tertulia filosófica (Biblio- tecas Públicas Municipales de Madrid).

—Poesía (9): recitales (BPE de Zaragoza), jornadas de poesía (Red de Bibliotecas Públicas de Mieres), certámenes de poesía infantil (BPE de Lugo) o actividades del tipo "Poesía para dibujar", que consiste en trasladar un poema a un dibujo (Servicio Municipal de Bibliotecas Públicas de San Javier).

-Teatro (9): la BPE de Valencia destaca la "Teatreca" expresión con la que alude al teatro en la biblioteca; la Biblioteca Pública de Moaña organiza la "Semana de teatro infantil"; y la Biblioteca Pública Municipal de Coaña "Gonzalo Anes" el "Mes del teatro".

-Feria del libro (5): en algunos casos se encargan de la organización de la feria del libro en su localidad, como la Biblioteca Pública Municipal de Burriana, que lo hace en colaboración con el Gabinete Psicopedagógico Municipal y participa a) como actividad principal, todos los niños de $1^{\circ}$ y $2^{\circ}$ de primaria visitan la biblioteca, donde se les explica brevemente su funcionamiento, $\mathrm{y}$ se realiza una pequeña animación relacionada con los libros; b) en un acto público realizado en el salón de actos de la casa de la cultura se entregan los diplomas a los niños que han participado en el taller de animación a la lectura "La ruta del llibre"; c) se procede al sorteo de varios lotes entre los niños y adultos que a lo largo del año han participado en la votación del libro que más les ha gustado; d) se coloca un stand en la calle, junto a las librerías de la localidad, en el que se exponen las últimas adquisiciones realizadas, intentando que el aspecto resulte atractivo a fin de que todas las personas que por allí pasean durante estos dos días se acerquen y puedan leer incluso en el mismo lugar los libros infantiles de la biblioteca, consiguiendo de este modo que muchas personas que no los conocían sepan de su existencia y se hagan socios.

\begin{tabular}{|l|c|c|}
\hline \multicolumn{1}{|c|}{ Actividad } & No de bibliotecas & $\begin{array}{c}\text { \% (sobre 183 bibliotecas } \\
\text { analizadas) }\end{array}$ \\
\hline Exposiciones & 54 & $29,5 \%$ \\
\hline Charlas y conferencias & 30 & $16,3 \%$ \\
\hline $\begin{array}{l}\text { Actividades varias con motivo de acontecimientos, } \\
\text { etc. }\end{array}$ & 24 & $13,1 \%$ \\
\hline Certámenes, concursos y premios & 23 & $12,5 \%$ \\
\hline Audiciones y actividades en torno a la música & 20 & $10,9 \%$ \\
\hline Cine, cine forum y otras actividades en torno al cine & 20 & $10,9 \%$ \\
\hline Presentación de libros & 20 & $10,9 \%$ \\
\hline Encuentros con autores & 13 & $7,1 \%$ \\
\hline Tertulias, coloquios y debates & 13 & $7,1 \%$ \\
\hline Poesía & 9 & $4,9 \%$ \\
\hline Teatro & 9 & $4,9 \%$ \\
\hline Feria del libro & 5 & $2,7 \%$ \\
\hline
\end{tabular}

Figura 1. Principales actividades culturales de las bibliotecas públicas españolas 


\section{¿Es la gestión de su colección electrónica}

\section{más complicada de lo que esperaba?}

\section{Nosotros le podemos ayudar.}

Conseguir el listado exacto de los títulos que ha suscrito y así poder gestionar correctamente el registro o solucionar los problemas de acceso a sus revistas y libros electrónicos, puede requerir más tiempo del que usted dispone.

Desde EBSCO gestionamos sus compras individuales o consorciadas de paquetes-e, incluyendo un listado exacto de las revistas y librose suscritos, facturas detalladas para facilitar las asignaciones de presupuestos y gestión personalizada de los informes de colecciones que le ayudarán en el desarrollo de su colección.

Nuestro equipo de servicio al cliente está a su disposición para ayudarle a resolver los problemas de acceso, los cambios en las direcciones IP, etc. Las herramientas de gestión de recursos-e de EBSCO han sido creadas con el fin de minimizar las tareas administrativas al tiempo que los usuarios maximizan sus resultados.

Ponemos toda nuestra información y experiencia a su disposición.

Contacte con su representante de EBSCO. 
-Monográficos (5): en torno a un tema de actualidad se elabora un dossier, una exposición virtual, o un apartado destacado en la sección "Agenda". Por ejemplo la información sobre Mozart disponible en la web de la Red Municipal de Bibliotecas de Sevilla.

-Actividades culturales (4): en algunos casos se anuncia la organización de actividades culturales pero no se especifica de qué tipo, como ocurre en la Biblioteca Pública Municipal de Armilla.

\section{Actividades de marketing}

— Visitas guiadas o en grupo (55): gozan de mucha tradición y están muy extendidas: Como ejemplo recogemos la explicación que aparece en la web de la Red de Bibliotecas Públicas de Ávila: las visitas guiadas están dirigidas especialmente a los colegios, permiten a la biblioteca mostrar su oferta tanto documental como de servicios, por lo que constituyen uno de los métodos de difusión y formación de usuarios más eficaces.

- Talleres (22): hacemos mención a los distintos talleres de animación a la lectura. Por ejemplo de internet y correo electrónico (Biblioteca Central de Igualada); de cómic, de encuadernación, de juegos de estrategia, de hierbas medicinales, etc. (Biblioteca "Joan Triadú" de Vic); coincidentes con fechas claves como Navidad, Carnaval, día del padre, etc., con actividades apropiadas para niños de diferentes edades (Red de Bibliotecas Municipales de Cartagena); taller de hip-hop, promovido por las Bibliotecas Públicas Municipales de Madrid y concebido como una propuesta para acercar a los jóvenes a las bibliotecas a través de la música y la creatividad, de una forma divertida y lúdica. No se necesita saber de melodías, tan sólo se precisa entusiasmo y tener algo que decir: los participantes crearán sus melodías y sus letras de tema libre. Piensan que con este taller se podrán crear poetas o músicos; de esta manera indirecta irán acercándose a la biblioteca y a la literatura. Aunque el taller está pensado para adolescentes, se acepta a todo aquel interesado en la música que quiera construir su propia rima, en todo aquel que tenga algo que decir. Quieren que en el taller además de aprender a hacer melodías y rima, aprendan a expresarse y lo hagan divirtiéndose, disfrutando con las melodías de hip-hop y descubran el alma poética y creativa que llevan dentro.

-Bibliopiscinas (10): esta ya clásica actividad la ofertan, entre otras, la Biblioteca Municipal de La Rambla, la Biblioteca Pública Municipal de La Pola de Lena "Menéndez Pidal”, o la Biblioteca Pública Municipal de Peñaranda de Bracamonte.

-Concursos de marcapáginas y pegatinas (8): se organiza un concurso para seleccionar marcapáginas que luego la biblioteca se encargará de difundir. Contamos en este caso con los ejemplos de la BPE de Almería "Francisco Villaespesa”, la Biblioteca Pública Municipal de Candás "Carlos González Posada” o la Red de Bibliotecas Públicas Municipales de Salamanca, en este caso también de pegatinas.

-Bebetecas (5): consiste en un servicio para bebés y sus padres. La Biblioteca Infantil de Can Butjosa incluye además un espacio $\mathrm{y}$ un fondo de libros, escogidos para las necesidades de los más pequeños y las de sus padres, el préstamo de estos libros, charlas periódicas sobre su uso y el de los cuentos, y un asesoramiento y atención constantes. Para la Biblioteca Municipal de la Palma de Cervelló tiene las finalidades siguientes: satisfacer las inquie- tudes y las aspiraciones de las familias con niños pequeños, iniciar y aproximar los niños a la cultura de los libros, fomentar desde pequeños el gusto por la lectura, iniciar a los padres y madres como agentes culturales activos de sus hijos, facilitar contactos y relaciones entre niños y familias a través de las actividades de la bebeteca, estimular el carácter afectivo entre los adultos y los niños, aprendizaje de hábitos (búsqueda de información, valorar los libros, comportamientos adecuados), y provocar la curiosidad en un entorno protegido. Se entiende como un servicio más dedicado a los niños más pequeños para los cuales la lectura consiste en ojear y escuchar lo que explican los padres.

La Biblioteca Pública Municipal de Coaña "Gonzalo Anes" utiliza la expresión "La Pequeteca", para referirse a un espacio de animación a la lectura con población infantil con edades comprendidas entre 3 y 6 años. Cada mes se dedica a unas jornadas con una temática distinta: cuentos para la Paz, el mes del teatro y la poesía, cuentos en red, un derecho infantil y un cuento, ecocuentos, jornadas de cuentos para reírse del miedo, etc.

- Programas y acciones en coordinación con otras instituciones (4): algunos centros destacan dentro de sus actos los que organizan con asociaciones culturales, colegios públicos, programas de voluntariado, etc. Es el caso de la Biblioteca Pública Municipal de Coaña "Gonzalo Anes" que las engloba dentro de las iniciativas de apertura de la biblioteca al exterior: de esta forma provocan que todas las personas tengan un mayor acceso a sus servicios mediante su descentralización.

-Bibliotecarios por un día (3): para explicar en qué consiste esta iniciativa sigamos la descripción 
que se recoge en la web de la Red de Bibliotecas Públicas de Mieres: desde noviembre a abril, las tardes de los martes, miércoles y jueves, una pareja de niños con edades comprendidas entre los 10 y 12 años (alumnos de $5^{\circ}$ y $6^{\circ}$ de primaria) atienden los préstamos y realizan todos los trabajos de organización de la biblioteca. Coincidiendo con el 23 de abril, día del libro, se celebra un acto de clausura en el que se entregan regalos y diplomas a los participantes. Se aúna la animación a la lectura y la formación de usuarios, a la vez se contribuye a la educación en valores de responsabilidad y supone un acercamiento de la biblioteca a la sociedad de la mano de los más jóvenes.

-Magia (3): la Red de Bibliotecas Públicas de Mieres dentro de las actividades de ocio anuncia "Escuela de Magia". También la $B P E$ de Lugo oferta la magia a los menores. Del mismo modo las Bibliotecas Públicas Municipales de Madrid incluyen en sus talleres uno denominado "Taller de la magia de los alfabetos".

-Programas en la radio (3): son varias las bibliotecas que utilizan este medio de comunicación como canal de promoción. Por ejemplo la Red de Bibliotecas Públicas de Mieres presenta el programa "La biblioteca en las ondas" en la radio local Parpayuela: todos los viernes a las 11:30 en la frecuencia 94.0 de FM. En este espacio se recomiendan libros, música, películas para niños, jóvenes y adultos, así como actos que se van realizando en la red a lo largo de la semana. Algo parecido sucede con la Biblioteca Pública Municipal de Castuera "Gonzalo Soubrier" y su "Programa de radio semanal" (viernes a las 12:00 horas) en $R a$ dio Zújar (107.4 FM), de carácter cultural e informativo; o con Las
Bibliotecas Municipales de Lor$c a$ y sus "Reseñas literarias en la radio", por medio de las cuales cada semana aconsejan un libro de la biblioteca.

-Actividades multiculturales o para inmigrantes (2): la $B P E$ de Almería "Francisco Villaespesa" promueve "Convive.con todos", es una semana multicultural que ha tenido como principal objetivo fomentar la sensibilización social acerca de los valores positivos de la multiculturalidad entre los niños y los jóvenes, para lo que se han desarrollado exposiciones, cuentacuentos, cine, charlas, talleres o guiñol. La Biblioteca Pública Central en Vigo ha organizado "Clase de supervivencia para adultos inmigrantes" y "Clases de apoyo para menores inmigrantes".

-Bibliojardines (2): un ejemplo destacado es la Red de Bibliotecas Municipales de Ávila que, durante los meses de julio y agosto, abre puntos de lectura con libros para todas las edades que se distribuyen por los lugares de ocio más característicos del verano, entre ellos los jardines.

— "Para conocer la ciudad" (2): organizada por la Red de Bibliotecas Públicas Municipales de Sevilla con la cual se conoce la historia y nombres de las calles.

- Tertulias o conversaciones en inglés (2): las celebra la Biblioteca Pública de Ciudad Real. Los participantes, coordinados por una nativa inglesa, y durante una hora a la semana, charlan en inglés sobre diferentes temas con lo que es posible practicar y mejorar los conocimientos previos sobre el idioma. La Biblioteca Pública Central, Vigo, oferta la actividad "Conversación en inglés" que consiste en grupos de debate de temas de actualidad para adultos.

- Tertulias literarias virtuales de escritores (2): anunciadas en su web por la BPE de Sevilla.

- "Ven y conoce tu biblioteca" (2): en la BPE de Sevilla y en la Biblioteca Pública Municipal de San Martín del Rey Aurelio.

- Concurso para la mascota de la biblioteca: tiene como meta la elección del diseño de una mascota y ha sido localizado en la Biblioteca Pública Municipal de Bembibre.

— “Jóvenes contra la violencia": es una idea de la Red Municipal de Bibliotecas de Sevilla para promover valores que ayuden a erradicar las prácticas violentas.

— "Noches en la biblioteca": la Red de Bibliotecas Públicas de Mieres abre en horas nocturnas y brinda actos culturales y de entretenimiento.

— "Biblioviaje": está planificada por la Red de Bibliotecas Públicas de Mieres en dos modalidades, una "Biblioviaje. Aventura", un barco espera para iniciar una travesía a la isla del tesoro de la mano del Pirata Malapata, los usuarios viajarán como bucaneros a una isla muy especial donde deben aprender a ser piratas valientes y arrojados, buscarán un tesoro y aprenderán todo sobre la supervivencia en una isla. La otra variante se denomina "Biblioviaje. El misterio", en este caso un tren espera a los usuarios para iniciar una aventura con muchos enigmas que resolver: sale de la Biblioteca de Turón y llega directamente a Ratonia donde $\mathrm{Ge}$ rónimo Stilton espera para llevar a los participantes a un tenebroso castillo donde aprenderán a ser buenos detectives y resolver un gran misterio.

—Juegos de mesa: La Biblioteca "Can Torró", de Alcúdia, propone a los usuarios que presenten su carnet para utilizar juegos de mesa como ajedrez, parchís, ordenador infantil, etc. 
— "Biblioteca de la memoria": actividad impartida en la Biblioteca Central de Igualada que consiste en recoger y conservar recuerdos de la vida cotidiana a través de la historia oral. Los interesados en aportar sus experiencias y recuerdos lo solicitan y un voluntario se hace cargo de realizar la entrevista.

- Semanas literarias: como la organizada por la Biblioteca de Moià, a lo largo de la cual se invita a personas relacionadas con el mundo de la industria literaria (editores, escritores, ilustradores, etc.).

—El rincón del juego: la Biblioteca del Mil-lenari, de Sant Cugat del Vallés, ofrece a los usuarios de menor edad, todos los viernes y también en época de vacaciones de verano o de Navidades, ir a jugar a ella. Encontrarán juegos educativos para aprender jugando con una monitora que ayuda en el manejo de los mismos.

- Club de los deberes: esta iniciativa se debe también a la Biblioteca del Mil-lenari para ayudar a los niños a integrar la biblioteca en su dinámica sociocultural. Intenta mejorar la comprensión lectora, usar diccionarios, etc.
-Encuentros de lectura: la Biblioteca Infantil de Can Butjosa organiza trimestralmente encuentros dedicados a los maestros para hablar de lectura, uso de la biblioteca, visitas didácticas, etc.

— "Apadrinamiento": puesta en práctica también por la Biblioteca Infantil de Can Butjosa, consistido en ampliar la atención hacia los niños que llegan a la biblioteca buscando precisamente atención y afecto además de información y conocimientos. De ello se encargan los lectores veteranos que en su día recibieron las atenciones de la biblioteca y por lo tanto son capaces de transmitirlas. Previamente se organizan cursos de voluntariado.

— "Aventura de llegir": diseñada por las Bibliotecas de Barcelona. Es una campaña que promueve el reconocimiento de la biblioteca pública como un equipamiento cultural. Entre los principales objetivos figura dar a conocer a los ciudadanos los servicios que presta y fomentar y ampliar los hábitos lectores de los usuarios potenciales. Se realiza mensualmente desde el año 1994 y se tra- tan temas tanto de humanidades y artes como de ciencia y tecnología.

- "Portes enllà", en la Red de Bibliotecas de la Diputación de Barcelona. Se trata de un conjunto de servicios para acercar la lectura a lugares frecuentados y captar nuevos usuarios. Las bibliotecas seleccionan entre sus fondos las obras adecuadas y las trasladan al lugar escogido, un espacio próximo al ciudadano. El ejemplo más significativo son las bibliopiscinas y biblioplayas, como servicio que se realiza durante el verano para acercar la lectura a los usuarios que gozan de su tiempo libre. También utilizan biblioterrazas, biblioplazas, biblioparques, y biblioramblas. Otro servicio curioso es el bibliomercado, que lleva los servicios de biblioteca a los mercados municipales, y el bibliotren, que presenta un espacio de la biblioteca en la estación municipal ferroviaria.

- Karaoke: ofertado por la Biblioteca Pública Municipal de Villajoyosa "Cristóbal Zaragoza".

-Bibliocampamentos de verano: la Biblioteca Pública Central en

\begin{tabular}{|l|c|c|}
\hline \multicolumn{1}{|c|}{ Actividad } & No de bibliotecas & $\begin{array}{c}\text { \% (sobre 183 bibliotecas } \\
\text { analizadas) }\end{array}$ \\
\hline Visitas guiadas o en grupo & 55 & $30,0 \%$ \\
\hline Talleres & 22 & $12,0 \%$ \\
\hline Bibliopiscinas & 10 & $5,4 \%$ \\
\hline Concurso de marcapáginas y pegatinas & 8 & $4,3 \%$ \\
\hline Bebetecas & 5 & $2,7 \%$ \\
\hline $\begin{array}{l}\text { Programas y actividades en coordinación con otras } \\
\text { instituciones }\end{array}$ & 4 & $2,1 \%$ \\
\hline Bibliotecarios por un día & 3 & $1,6 \%$ \\
\hline Magia & 3 & $1,6 \%$ \\
\hline Programas en la radio & 3 & $1,6 \%$ \\
\hline
\end{tabular}

Figura 2. Principales actividades de marketing de las bibliotecas públicas españolas 
Vigo, para niños de 4 a 9 años; se persigue que los usuarios que frecuentan la biblioteca en estos días participen de trabajos manuales, cuentos y canciones.

— "Conócelos y aprende": bajo esta expresión, el Servicio Municipal de Bibliotecas Públicas de San Javier acerca la sabiduría y experiencias de los mayores a los más jóvenes. Se han realizado sesiones en las que preguntan a los mayores por episodios de sus vidas para, posteriormente, realizar un trabajo en sus centros educativos. Al final se elabora un dossier que se incorpora a la sección local de la biblioteca.

_"Biblioteca solidaria": la Biblioteca Pública Barañain, convencida de que con los libros podemos ayudar, organiza una campaña de recogida de libros usados para una $\mathrm{ONG}$.

\section{Recursos en línea}

- Novedades (45): las bibliotecas anuncian con frecuencia las obras adquiridas recientemente, una selección de libros que aconsejan, etc., bien en formato pdf o doc (Biblioteca Central de Igualada) para descargar, o en formato html (Biblioteca "Josep Roca Bros", de Abrera). En algunos casos las novedades se difunden a través de un boletín específico, como el de "Ratón de biblioteca" de la Biblioteca Pública Municipal de Peñaranda de Bracamonte o de "L'aparador" (el escaparate) de la Biblioteca Central de Terrassa.

- Logotipos (41): como muestra significativa presentamos los siguientes: la Red Municipal de Bibliotecas de Sevilla se promociona con un logotipo que incluye un símbolo de carácter icónico relacionado con la acción de escribir mediante la referencia realista de unas plumas y un trazo ilegible. Las Bibliotecas de Barcelona han diseñado un logotipo que incluye un símbolo de gran simplicidad abstracta, puede recordar un libro abierto o la inicial "B" tumbada, el diseño destaca en simetría y regularidad. En el logotipo de las Bibliotecas Públicas Municipales de Madrid atrae el símbolo icónico y alfabético que juega con el motivo del oso y el madroño, éste último se sustituye por las iniciales de la biblioteca en colores parchís, propios de la tendencia del informalismo cromático.

- Guías de lectura (34): constituyen el segundo recurso más extendido entre las bibliotecas consultadas. Como muestra destacamos: las Bibliotecas de Barcelona que presentan una sección específica denominada "Guías de lectura", recopilando todas las editadas por esta institución en formato pdf; el conjunto de guías de la Biblioteca Central de Terrassa: "Rutes de la biblioteca" (para el público adulto); "Segueix el fil de la infantil" (para los niños); "L'itinerari" (lista mensual de los materiales de un tema o de un autor que se pueden encontrar en el área general); "Menú del mes" (lista mensual de los materiales de un tema o de un autor que se pueden encontrar en el área infantil); "Tal com sona" (lista mensual de los CDs que de un autor, tema o género musical se pueden encontrar en el área de música y cine).

- Rincón infantil o bebeteca (13): es frecuente que en las webs de las BPs se dedique un lugar diferenciado para los niños con información y recursos específicos y diseñado con una estética adaptada a los usuarios; como ejemplos señalamos la sección "Rincón infantil" de las Bibliotecas Públicas del Estado de Málaga y de Sevilla o de las Bibliotecas de Barcelona. Con la expresión "Bebeteca" aparece en la Biblioteca Municipal de la Palma de Cervelló. Tres bibliotecas lo dedican al público infantil y juvenil: BPEs de Valladolid y de Málaga.

- Trípticos y folletos sobre los servicios, actividades, etc. (12): nos referimos a las bibliotecas que han colgado en su web este tipo de documentos reproducidos casi siempre en formato pdf. Así el Servicio Municipal de Bibliotecas de Córdoba, reproduce el folleto para difundir el servicio de préstamo; la Biblioteca Pública "Salvador Raurich", de Begur, el tríptico guía de la biblioteca; o la Biblioteca Municipal de Albaida el de la campaña de animación lectora.

- Formularios para visitas y otras actividades (9): las bibliotecas hacen posible que los usuarios soliciten vía web las visitas, o la participación en actividades, para lo que tienen disponibles unos formularios que los usuarios interesados deben rellenar y enviar. Para ver esos formularios podemos acudir a las webs de la BPE de Almería "Francisco Villaespesa"; de la Red de Bibliotecas Públicas de Andalucía o de la Biblioteca Pública Municipal de Avilés. Ejemplo de formulario para solicitar el envío por e-mail del calendario de actividades es el de la $B P E$ de Lérida y de reservas de instalaciones el de la BPE de Mérida "Jesús Delgado Valhondo".

- Revistas o boletines de las bibliotecas (6): algunos centros que cuentan con una publicación de este tipo lo tienen disponible a texto completo en la web. Es el caso de la BPE "Can Sales" de Palma de Mallorca; la Biblioteca "Joan Triadú", de Vic; o de la BPE de Badajoz "Bartolomé J. Gallardo".

- Reproducción de marcapáginas (4): nos referimos a las bibliotecas que nos muestran a través de la web aquellos que han diseñado los usuarios y que en la mayoría de las ocasiones han participado en un concurso previo. Tenemos la posibilidad de copiarlos o imprimirlos. Valgan de muestra los casos de la Biblioteca Pública Municipal de Grado "Valentín Andrés Álvarez" y los de la Biblioteca Municipal de Cassà de la Selva.

- Sección profesional

(4): 
bajo esta denominación encontramos disponibles documentos a texto completo especializados para bibliotecarios en la Biblioteca Pública Municipal de Peñaranda de Bracamonte; o en la BPE de Ciudad Real. La BPE de Tarragona tiene colgado un documento que explica los centros de interés y un manual de procedimientos.

- Planos de distribución de fondos y espacios (3): para facilitar el conocimiento del edificio y como forma de promoción de sus espacios se reproducen los planos de los mismos. Entre los ejemplos disponibles citamos la Biblioteca Pública de Roses "Jaume Vicens Vives", la Biblioteca Central de Terrassa y la Biblioteca Infantil de Can Butjosa. Este último caso consiste en una ilustración de los espacios de la biblioteca con dibujos infantiles, propuesta como visita virtual.

- Guías del inmigrante (2): diseñadas para ayudar a la integración de la población inmigrante. Véase como ejemplo la BPE de Oviedo.

- Catálogos de exposiciones (2): mostrados en las webs de la BPE de Zamora; o la Red de Bibliotecas de Alcoy.
- Textos informativos sobre autores locales (2): este recurso lo hemos visto en la Biblioteca Municipal de La Rambla. Muy semejante es la sección "Escritores pravianos" de la Biblioteca Pública Municipal de Pravia "Antón de la Braña", que incluye una reseña y fotografía de cada escritor.

— "El granadino del mes": iniciativa de la BPE de Granada que muestra periódicamente la fotografía y datos biográficos de un personaje de la ciudad.

— "Sección de inéditos": la $B P E$ de Granada dedica un espacio de su web para difundir trabajos inéditos de autores granadinos.

— "Sevilla literaria": la Red Municipal de Bibliotecas de Sevilla ha diseñado un recurso que permite realizar un recorrido virtual por las calles de la ciudad estableciendo su relación con la literatura.

— "Así era": la Biblioteca Pública Municipal de Pravia "Antón de la Braña” recoge en la sección de este nombre fotos antiguas de la localidad.

- Cartel de silencio: en la web de la Red de Bibliotecas Públicas del Ayuntamiento de Oviedo está disponible en formato pdf el cartel para guardar silencio.

- Buzón para devoluciones: para facilitar la vuelta de los libros fuera del horario de apertura de la biblioteca (práctica habitual en establecimientos de carácter comercial como los vídeoclubs) la $B P E$ de Burgos ha instalado un buzón de este tipo y lo promociona a través de la web.

- Álbum de firmas: la Biblioteca Infantil de Can Butjosa ha introducido en su web un álbum de firmas de los personajes y escritores que la han visitado.

- Formulario para seleccionar el tipo de actividad: la Red de Bibliotecas de la Diputación de Barcelona para facilitar la búsqueda de actividades que organiza la red ha diseñado un formulario específico.

— "El rincón del lector": bajo esta denominación las Bibliotecas Públicas Municipales de Madrid han dispuesto un foro de opinión sobre un tema de actualidad.

- "Gente mayor en Tarragona": la BPE de Tarragona dispone de una recopilación de recursos

\begin{tabular}{|l|c|c|}
\hline \multicolumn{1}{|c|}{ Denominación del recurso } & No de bibliotecas & $\begin{array}{c}\text { (sobre 183 bibliotecas } \\
\text { analizadas) }\end{array}$ \\
\hline Novedades & 45 & $24,5 \%$ \\
\hline Logotipos & 41 & $22,4 \%$ \\
\hline Guías de lectura & 34 & $7,1 \%$ \\
\hline Rincón infantil o bebeteca & 13 & $6,5 \%$ \\
\hline Trípticos y folletos sobre servicios, etc. & 12 & $4,9 \%$ \\
\hline Formularios para visitas, etc. & 9 & $3,2 \%$ \\
\hline Revistas o boletines & 6 & $2,1 \%$ \\
\hline Reproducción de marcapáginas & 4 & $2,1 \%$ \\
\hline Sección profesional & 4 & $1,6 \%$ \\
\hline Planos de distribución de fondos y espacios & 3 & \\
\hline
\end{tabular}

Figura 3. Principales recursos en línea culturales y de marketing de las bibliotecas públicas españolas 
para las personas mayores ubicados en la ciudad y en la biblioteca.

\section{"Las bibliotecas optan por el juego y la diversión como herramientas peda- gógicas y de motivación"}

\section{Conclusiones}

Los resultados de este trabajo reflejan que el modelo clásico de biblioteca pública está cambiando, y fruto de esta transformación es la elaboración de actividades y recursos como los analizados, que tienden a convertir estos centros en lugares de formación y en una alternativa al ocio, bajo un prisma que aúna el entretenimiento con los aspectos culturales y formativos.

A través de los datos recopilados se intuye que las bibliotecas están optando por el juego y la diversión como herramientas pedagógicas y de motivación, así como por fomentar su uso como lugar de socialización y de convivencia.

El marketing cuantitativamente está menos presente (sólo 3 tipos de acciones superan su presencia en más del 5\% de las bibliotecas analizadas) en comparación con las culturales $(9$ clases están presentes en más del 5\% de los centros consultados), pero hay una mayor variedad (32 distintas, frente a 13 culturales), son más innovadoras y reflejan las tendencias comentadas en la bibliografía especializada.

Si comparamos los tipos de acciones establecidas en la literatura previa analizada (citadas al principio del trabajo), observamos lo siguiente:

De las actuaciones para "promoción y comercialización" aconsejadas por las Directrices IFLA/ Unesco para el desarrollo del servicio de bibliotecas públicas (2002) no aparecen en los sitios web ana- lizados: "grupos de amigos de la biblioteca" y "campañas de recaudación de fondos". Y entre las que gozan de una mayor presencia están las exposiciones, las "webs de la biblioteca", y las "celebraciones de años especiales y aniversarios".

De las Pautas Calimera de buena práctica (2005) y las Pautas Pulman (2002) no se comprueba a través de los datos recogidos si se realiza "el envío de publicidad por correo a los posibles usuarios"; y tampoco las "visitas a empresas y asociaciones de la localidad", aunque sí que están muy extendidas las visitas a los colegios y centros educativos. Las prácticas mejor representadas, de entre las que ambos textos indican, son: "publicar listados de fondos bibliográficos" y "exponer información detallada en el servidor web de la biblioteca".

Las actividades culturales recomendadas por las Pautas sobre los servicios de las bibliotecas públicas (2002) y por García Rodríguez (1996) están todas presentes. Igual sucede con la propuesta de Quílez (2002), a excepción de la "publicidad genérica", difícil de comprobar de manera precisa a través de las webs.

Finalmente, de la sistematización de Massísimo (2005) tienen muy escasa presencia el bibliomercado y el bibliometro. De los productos de promoción están bien representados los logotipos, los marcapáginas y los folletos, mientras que no tenemos noticia sobre carteles o camisetas.

En un sentido amplio las actividades que tradicionalmente se han denominado culturales (exposiciones, mesas redondas y debates, presentaciones de obras o colecciones, representaciones teatrales, audiciones musicales, proyecciones cinematográficas, conferencias) tienen una dimensión de promoción y marketing, pues ayudan a mejorar la imagen de la biblioteca, a que se hable de ella, que sea noticia y a que los usuarios acudan con nuevos intereses; y todo ello aunque en muchas ocasiones no formen parte de una campaña específica de promoción. Por otro lado el marketing va más allá de la promoción, del dar a conocer la biblioteca y hacerla atractiva, pues -como afirma Gómez Hernández (2002)- supone también convencer a los usuarios de utilizar los servicios, y sobre todo mantenerse unidos a ellos para conocer sus expectativas y ajustar la biblioteca a las necesidades detectadas.

Nuestras bibliotecas públicas cada vez más funcionan en un contexto híbrido, con una doble dimensión física y virtual; en este trabajo lo hemos constatado en los recursos virtuales recopilados.

De todas las páginas y sitios web de bibliotecas consultadas sobresalen por la cantidad y variedad de actividades organizadas, así como por la detallada información que ofrecen al respecto las siguientes:

- Bibliotecas Públicas Municipales de Madrid.

- Bibliotecas de Barcelona.

- Red de Bibliotecas Públicas de Mieres.

- Biblioteca Pública Municipal de Peñaranda de Bracamonte.

- Biblioteca Infantil de Can Butjosa.

— Red de Bibliotecas Públicas Municipales de Cartagena.

- BPE de Guadalajara.

- Red de Bibliotecas de la Diputación de Barcelona.

— Red de Bibliotecas Municipales de Bilbao.

\section{Nota}

1. Las visitas a las webs de las bibliotecas se han realizado durante los meses de mayo y junio de 2006. 
http://www.bibliotecaspublicas.es/index.jsp?pw $=0$

\section{Bibliografía}

Caballero Garrido, A. "La extensión bibliotecaria”. En: Magán Wals, J. A. (coord.). Tratado básico de biblioteconomía. $4^{\mathrm{a}}$ ed. Madrid: Universidad Complutense, 2002, pp. 381-396. Isbn 84-7491-670-4.

Directrices IFLA/Unesco para el desarrollo del servicio de bibliotecas públicas. Madrid: Secretaría General Técnica, Subdirección General de Información y Publicaciones, 2002. ISBN 84369-3640-x.

García Rodríguez, A. "Actividades culturales en la biblioteca". En: Orera Orera, L. (ed.) $M a$ nual de biblioteconomía. Madrid: Síntesis, 1996, pp. 289-296. ISBN 84-7738-363-4.

Gómez Hernández, J. A. Biblioteconomía general y aplicada: conceptos básicos de gestión de bibliotecas. Murcia: D. M., 1997. ISBN 8489820-37-6.

Gómez Hernández, J. A. Gestión de bibliotecas. Murcia: D. M., 2002. ISBN 84-8425-262-0.

Massísimo i Sánchez de Boado, À. "Màrqueting bibliotecari: una realitat en desenvolupament". En: Item: revista de biblioteconomia i documentació, 2005, gener-desembre, v. 39, pp. 5-25.

Norman, O. Gene. "Marketing library and information services: an annotated guide to recent trends and developments". En: Reference services review, 1989, Spring, v. 17, n. 1, pp. 43-64.

Pautas Calimera de buena práctica, 2005. Consultado en: 29-10-06.

http://www.calimera.org/Countries/Spain.aspx Pautas Pulman: Public libraries mobilising advanced networks, 2002. Consultado en: 29-1006.

http://www.pulmanweb.org/DGMs/DGMs.htm Pautas sobre los servicios de las bibliotecas públicas. Consultado en: 25-06-06. http://travesia.mcu.es/documentos/pautas_servicios.pdf

Quílez Simón, P. "Metodologías para el análisis de las actividades culturales en el ámbito de la biblioteca pública". En: I Congreso nacional de bibliotecas públicas, 2002. Valencia.

http://travesia.mcu.es/documentos/actas/com 265.pdf

José Luis Herrera Morillas, Margarita Pérez Pulido, Universidad de Extremadura, Facultad de Biblioteconomía y Documentación, Plazuela de Ibn Marwan, s/n (Antiguo Hospital Militar), 06071 Badajoz.

jlhermor@alcazaba.unex.es marga@alcazaba.unex.es

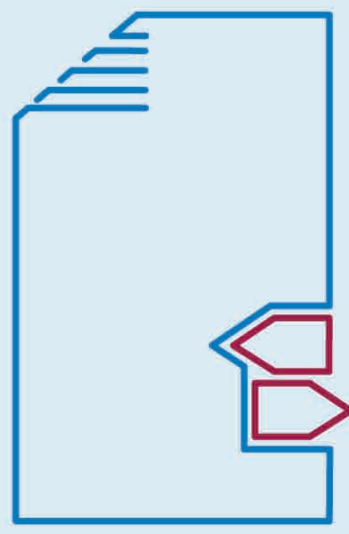

SIBADOC

Soluciones integrales en informática documental y servicios de información

- Automatización de bibliotecas, archivos y centros de documentación

- Gestión de documentos electrónicos

- Externalización de servicios (catalogación, digitalización, organización de archivos, etc.)

- Formación en tecnologías de la información y la documentación

- Desarrollo y distribución de aplicaciones en tecnología .NET

\section{EMPRESA OUE HA REALYAAO LA EDICIÓN DIGIYAL EN CD-ROM DE "EDUCACIÓN Y BIBLIO'TECA"}

\section{Preparada para todo aquello que tu centro de información quiera emprender}

C/ Pedro Teixeira, 9, esc. dcha. 3D - 28020 Madrid - 915983584 / C/ Nicasio Gallego, 4 - 5 dcha. 28010 Madrid - 670910841

sibadoc@sibadoc.com

www.sibadoc.com 


\section{LA RED」SA DEL MUNDO DE LAS BIBLIOTECAS}

abiblioteca EBibliotica.

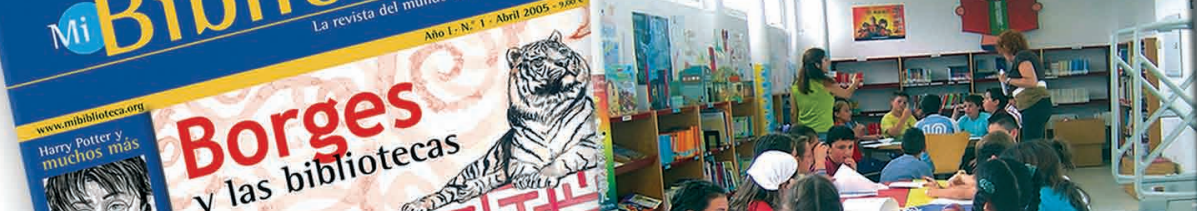

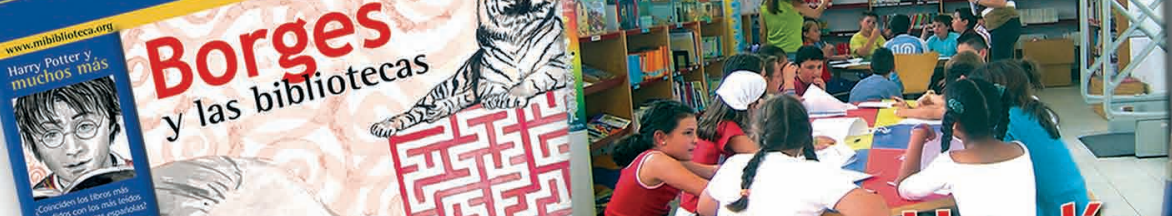
Biblioteca

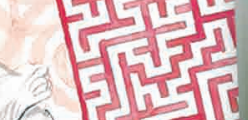
(2) $12 \sqrt{2}$

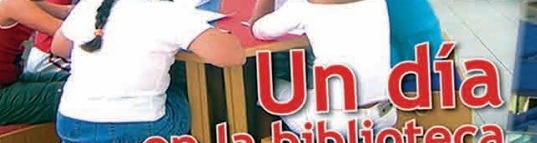
Biblioteca

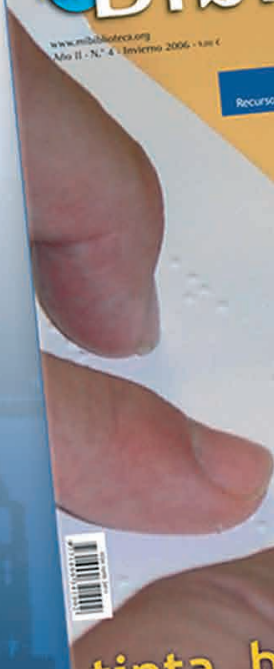

S Un: tugar ileno

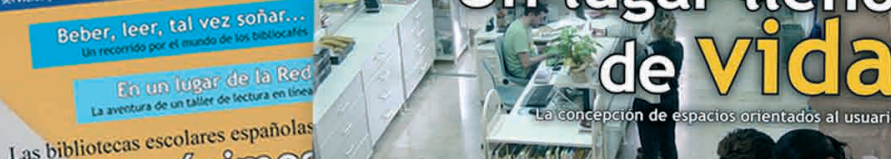

Wiblioteca

Alleyentidiria

tinta, braille y sono

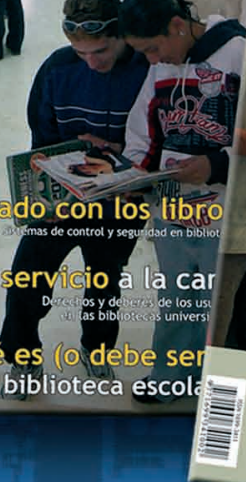

Bibliotecas

en la cárcell (a) 